\title{
Análise da produção de artigos científicos brasileiros sobre o Teste de Zulliger
}

\author{
Lucila Moraes Cardoso \\ Gabriel Vitor Acioly Gomes \\ Fabio Pinheiro Pacheco \\ João Lucas Dias-Viana
}

\section{RESUMO}

Este estudo objetiva verificar como estão sendo conduzidas as pesquisas sobre o método de Zulliger após 2003. Foi feita uma busca na Biblioteca Virtual de Saúde, consultando todos os artigos completos disponibilizados na base bibliográfica Index Psi Periódicos TécnicoCientíficos, utilizando o descritor "Teste de Zulliger". Obtiveram-se 20 artigos que foram analisados considerando informações sobre autoria; periódico e ano da publicação e sistema interpretativo usado. Além disso, foi feita uma análise descritiva dos objetivos, métodos e resultados dos artigos. Prevaleceram os estudos que visavam buscar as qualidades psicométricas do Zulliger, em especial, de evidências de validade. Conclui-se que há uma preocupação com as qualidades psicométricas do Zulliger, mas ainda é preciso aumentar a frequência com que esses estudos ocorrem.

Palavras-chave: avaliação psicológica; métodos projetivos; técnicas de autoexpressão; Teste de Zulliger.

\section{ABSTRACT}

\section{Analysis of the production of Brazilian scientific articles about Zulliger test}

This study aims to describe the current research on the Zulliger method since 2003. We searched in the Bireme's Virtual Health Library, consulting all full articles available on the bibliographic database Index Psi, using "Zulliger Test" as keywords. Twenty articles were obtained, which were analyzed according to the author, journal, year of publication and interpretation system used. Most of the studies aimed at psychometric qualities of Zulliger, namely evidence of validity. We conclude that there is a concern about the psychometric qualities of Zulliger, but the frequency of which these studies occur should increase.

Keywords: psychological assessment; projective methods; self-expression techniques; Zulliger test.

A avaliação psicológica é um processo complexo, que envolve tanto o segmento da Psicologia voltado para a criação e estudos dos instrumentos e métodos psicológicos quanto um processo no qual há coleta de dados sobre indivíduos e/ou contextos, buscando, sistematicamente, conhecimento sobre o funcionamento psicológico das pessoas, a fim de orientá-las em suas ações e decisões (Joly, Silva, Nunes, \& Souza, 2007; Primi, 2010). Na perspectiva de assinalar o percurso histórico da área de Avaliação Psicológica, Primi (2010) e Noronha e Reppold (2010) apontaram os principais avanços ocorridos nos últimos anos. Esses progressos podem ser exemplificados pela criação das associações de representação da área, a implantação do Programa de Pós-Graduação em Avaliação Psicológica da Universidade São Francisco, a criação e circulação de periódicos específicos e o aumento das produções científicas na área.

\section{Sobre os Autores}

L. M. C.

orcid.org/0000-0002-8890-9352 Universidade Estadual do Ceará

- Fortaleza, CE

lucila.cardoso@uece.br

\section{G. V. A. G.}

orcid.org/0000-0001-6565-3094

Universidade Estadual do Ceará

- Fortaleza, CE

gabrigom93@hotmail.com

\section{F. P. P.}

orcid.org/0000-0003-4139-1506

Universidade Estadual do Ceará

- Fortaleza, CE

pfabiopinheiro@gmail.com

\section{J. L. D. V.}

orcid.org/0000-0002-7626-3937 Universidade Estadual do Ceará

- Fortaleza, CE

jo_lukas@hotmail.com

\section{Direitos Autorais}

Este é um artigo de acesso aberto e pode ser reproduzido livremente, distribuído, transmitido ou modificado, por qualquer pessoa desde que usado sem fins comerciais. 0 trabalho é disponibilizado sob a licença Creative Commons CCBY-NC.

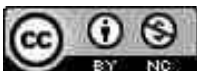




\section{H INERACÄOEM LF PSICOLOGIA}

Os avanços da avaliação psicológica foram além do âmbito acadêmico, interferindo diretamente na prática dos psicólogos. O Conselho Federal de Psicologia (CFP), por meio da resolução CFP n 009/2018, estabeleceu critérios mínimos e obrigatórios para a elaboração de testes psicológicos no Brasil, que atestam o caráter científico do instrumento e criou o Sistema de Avaliação dos Testes Psicológicos (SATEPSI). Dentre os requisitos necessários para que um instrumento possa ser considerado adequado estão os critérios de normatização, padronização, precisão e evidências de validade, conhecidos como qualidades psicométricas (Serafıni, Budzyn, \& Fonseca, 2017).

0 conceito de validade diz respeito ao grau em que o teste avalia o que se propõe a medir; conjunto de evidências que dão garantia científica à interpretação dos resultados obtidos no teste e atestam seu uso. A precisão ou fidedignidade refere-se à consistência e manutenção dos resultados obtidos em diferentes momentos de avaliação, utilizando o mesmo teste ou formas equivalentes (Pasquali, 2010). A padronização refere-se à uniformidade de procedimentos de aplicação e avaliação e a normatização aos padrões de avaliação e interpretação dos resultados de acordo com normas padronizadas (Miguel, 2017).

Em relação às pesquisas desenvolvidas na área, Noronha e Alchieri (2002) já assinalavam que o principal foco de estudos na avaliação psicológica voltava-se à busca das qualidades psicométricas dos instrumentos e sua construção. Ainda assim, as pesquisas eram incipientes, tornando-se necessário incentivar a construção e adaptação de mais instrumentos com qualidades psicométricas e processos avaliativos para diferentes contextos, conforme apontaram Hutz e Bandeira (2003).

Na perspectiva de analisar como estavam sendo conduzidas as pesquisas na área de avaliação psicológica, Joly et al. (2007) realizaram uma análise dos resumos de 934 painéis apresentados nos Congressos Nacionais de Avaliação Psicológica realizados em 2003, 2005 e 2007. Os pesquisadores observaram um aumento progressivo de trabalhos do congresso de 2003 (total de 263) para o de 2007 (total de 346), notando-se também o aumento da porcentagem de trabalho que envolvesse o estudo das qualidades psicométricas (35\% em 2003 para 42,5\% em 2007) em detrimento dos estudos de aplicação (47,1\% em 2003 para 24,9\% em 2007) dos instrumentos.

No mesmo sentido Joly, Teixeira, Berberian e Andrade (2010) analisaram 141 resumos de teses e dissertações disponíveis na Biblioteca Virtual em Saúde do Brasil (BVS-Psi Brasil) e que tinham as palavras-chave avaliação psicológica, psicometria, validade, precisão e testes psicológicos disponíveis. Obtiveram uma porcentagem maior de dissertações de mestrado (54,6\%), seguido pelas teses de doutorado (43,3\%) e de pós-doutorado (2,1\%). Destes, $80,14 \%$ foram defendidos em Universidades estabelecidas na região Sudeste do País, $19,15 \%$ no sul e $0,71 \%$, no Nordeste. A maioria dos trabalhos $(60,3 \%)$ buscou por parâmetros psicométricos e $27,7 \%$ tinham a finalidade de utilizar os testes para validar protocolos de intervenção, descrever habilidades específicas e estudar relações entre variáveis.

Dentre os diversos instrumentos de avaliação, há os chamados Métodos Projetivos. Nos últimos anos, há um debate internacional sobre qual seria a melhor nomenclatura a ser dada para esse conjunto de técnicas (Rietzler, 2006; Meyer \& Kurtz, 2006; Miguel, 2014, entre outros). Cardoso e VillemorAmaral (2017) defenderam que, para além da escolha da nomenclatura em si, é importante que se amplie a compreensão sobre as polêmicas que envolvem as particularidades atribuídas a esse conjunto de métodos. Como essas questões não são o foco do presente artigo e diante da ausência na literatura, até o momento, de um termo que consiga abarcar as peculiaridades envolvidas nesses instrumentos, optamos por manter o termo Método Projetivo, embora se reconheça que alguns pesquisadores da área têm recomendado o termo Técnica de autoexpressão.

Os métodos projetivos são ferramentas importantes que auxiliam na identificação de traços da personalidade e sintomas de quadros psicopatológicos (Fensterseifer \& Werlang, 2008). Esses instrumentos possuem características bastante peculiares, entre eles, o fato dos estímulos serem pouco estruturados, as orientações fornecidas aos examinandos serem breves e gerais e não haver uma noção de respostas consideradas certas ou erradas.

Essas particularidades são fundamentais para assegurar a dimensão idiográfica desses métodos, possibilitando assim a manifestação de aspectos únicos e próprios do funcionamento interno do indivíduo avaliado (Fensterseifer \& Werlang, 2008). Essa dimensão idiográfica é defendida como uma das principais vantagens para o uso desses métodos em situações que se tenha o objetivo de conhecer aspectos da dinâmica de personalidade da pessoa avaliada, sendo também responsável pelas polêmicas à aplicabilidade das exigências relativas às propriedades psicométricas desses instrumentos (Villemor-Amaral \& Pasqualini - Casado, 2006).

Um método projetivo que vem apresentando crescente número de estudos é o teste de Zulliger, um instrumento constituído por três pranchas com manchas de tintas, que são considerados estímulos não-estruturados. Esse instrumento fornece diversas informações, entre elas aspectos do funcionamento cognitivo e afetivo do examinando, percepção, controle emocional e relações interpessoais (VillemorAmaral \& Primi, 2009). No Brasil, o Zulliger possui duas ver- 


\section{H NTERAC̄OOEM IF PSICOLOGIA}

Lucila Moraees Cardoso, Gabriel Vitor Acioly Gomes,

Fabio Pinheiro Pacheco e João Lucas Dias Viana sões com parecer favorável para uso, sendo o Sistema Compreensivo, que só pode ser usado com administração individual (Villemor-Amaral \& Primi, 2009), e o Sistema Klopfer, que inicialmente foi padronizado à administração coletiva (Vaz, 1998) e recentemente atualizado com estudos que possibilitam o uso coletivo ou individual (Vaz \& Alchieri, 2016).

$\mathrm{O}$ teste de Zulliger tem sido usado em diferentes estudos (Ferreira \& Villemor-Amaral, 2005; Villemor-Amaral, Machado, \& Noronha, 2009; Villemor-Amaral \& Machado, 2011; Franco \& Villemor-Amaral, 2012; Franco \& Villemor-Amaral, 2012b; Grazziotin \& Scortegagna, 2012; Villemor-Amaral \& Cardoso, 2012; Grazziotin \& Scortegagna, 2013; Villemor-Amaral \& Quirino, 2013; Tavella \& Villemor-Amaral, 2014; Grazziotin \& Scortegagna, 2016; Villemor-Amaral \& Vieira, 2016; Villemor-Amaral, Pavan, Tavella, Cardoso, \& Biasi, 2016; Gregoleti \& Scortegagna, 2017), fazendo-se interessante compreender como estão sendo conduzidas as pesquisas sobre as qualidades psicométricas deste instrumento por meio de uma revisão de literatura. Esta modalidade de estudo possibilita focalizar aspectos amplos das produções científicas de uma determinada área, contribuindo para a análise do saberfazer-poder científico (Joly et al., 2010).

Recentemente, Grazziotin e Scortegagna (2016b) publicaram um artigo de revisão de literatura para verificar os estudos publicados com o uso do Zulliger no Brasil considerando as faixas etárias e os objetivos. Entre as indicações de pesquisas futuras, as autoras sugeriram estender a investigação feita abordando as regiões brasileiras em que os estudos foram conduzidos, bem como relacionar as revistas em que foram publicados os artigos. Essa sugestão foi adotada como uma das vertentes de análise dos resultados da presente revisão de literatura.

Desta maneira, assumindo uma perspectiva diferente, mas complementar ao estudo de Grazziotin e Scortegagna (2016b), objetivou-se nesta pesquisa analisar os artigos sobre as qualidades psicométricas do teste de Zulliger disponíveis na base bibliográfica Index Psi Periódicos Técnico-Científicos da Biblioteca Virtual de Saúde - Psicologia Brasil (BVS-Psi Brasil). Assim, espera-se que o presente artigo possa colaborar à compreensão dos estudos desenvolvidos com o Zulliger com amostras da população brasileira no período de 2003 a 2017.

\section{MÉTODO}

\section{Composição da amostra e material}

Os artigos analisados no presente trabalho foram obtidos por meio de um levantamento na Biblioteca Virtual de Saúde - Psicologia Brasil (BVS-Psi Brasil). Foram considerados os artigos completos constantes na base bibliográfica Index Psi Periódicos Técnico-Científicos.

Primeiramente, foi feita uma busca sobre a terminologia em Psicologia mais indicada. Assim, foi digitada a palavra "Zulliger" e o descritor sugerido para uso foi "teste de Zulliger". Usando esse termo, foi possível obter 33 referências, sendo que 16 foram excluídas deste trabalho. Os motivos da exclusão foram o fato do trabalho não estar disponibilizado na íntegra em bases de acesso livre $(n=11)$, por citarem o Zulliger no decorrer do trabalho sem terem feito uso do instrumento $(n=2)$, pelo artigo ter aparecido na busca porque um dos autores tinha o sobrenome Zulliger, mas o trabalho não tinha relação com o instrumento $(n=1)$ e pelas pesquisas terem sido feitas com amostras exclusivamente de Portugal ou da França $(n=2)$. Assim, para esta revisão da literatura foram considerados 20 artigos completos publicados entre 2003 e 2017.

\section{Procedimento}

Após a seleção dos 20 artigos, os mesmos foram lidos e quantificados de acordo com três categorias criadas, a saber, Informações sobre a autoria e instituição de vínculo dos primeiros autores; Periódico e ano da publicação dos artigos; e Informações sobre o sistema interpretativo do teste de Zulliger. Todas essas categorias foram compostas por mais de um indicador.

$\mathrm{Na}$ categoria sobre as informações relacionadas à autoria dos artigos considerou-se a quantidade de autores, o sexo e a titulação do primeiro autor e as informações sobre a instituição de origem do primeiro autor. A segunda categoria constituiu-se de informações sobre o periódico e o ano da publicação e, por fim, a terceira categoria quantificou dados sobre o sistema interpretativo do Zulliger em relação à origem do primeiro autor. Posteriormente, foi feita uma breve descrição dos objetivos, métodos e resultados de cada um dos estudos realizados com o Zulliger.

\section{RESULTADOS}

Após fazer o levantamento dos 20 artigos (Tabela 1), verificou-se que, dos estudos com o Zulliger pelo sistema interpretativo Klopfer (Vaz, 1998), dois tinham objetivos relacionados ao uso desse instrumento em contextos específicos e um buscava por evidências de validade para uso do referido teste com participantes adultos. No mesmo sentido, ao analisar os estudos com o Zulliger pelo Sistema Compreensivo (ZSC) de Villemor-Amaral e Primi (2009), verificou-se que todos visavam analisar as qualidades psicométricas do instrumento, sendo um de precisão e 13 de evidências de va- 


\section{H NTERAC̄öEM से PSICOLOGIA}

lidade. Destes, oito destinavam-se a pesquisar as evidências de validade para o uso com adultos, quatro para uso com crianças e um estudo com idosos. Além disso, foram encontra- dos dois estudos usando um manual publicado na Suiça em 1957 e um estudo usando um manual publicado na Argentina em 1970.

\begin{tabular}{|c|c|c|c|c|}
\hline $\begin{array}{c}\text { Sistema } \\
\text { Interpretativo }\end{array}$ & Autoria & Periódico & Objetivo & Part. \\
\hline Klopfer & Montes \& Vaz (2003) & $\begin{array}{c}\text { Psicologia: Teoria e } \\
\text { Pesquisa }\end{array}$ & $\begin{array}{l}\text { Investigar afetividade } \\
\text { ou cognição }\end{array}$ & Adultos \\
\hline Klopfer & Angelini \& Oliveira (2003) & $\begin{array}{c}\text { PSIC - Revista de Psicologia } \\
\text { da Vetor Editora }\end{array}$ & $\begin{array}{l}\text { Evidências de } \\
\text { validade }\end{array}$ & Adultos \\
\hline Klopfer & Rodrigues \& Alchieri (2009) & Psico-USF & $\begin{array}{l}\text { Investigar afetividade } \\
\text { e cognição }\end{array}$ & $\begin{array}{l}\text { Crianças } \\
\text { e adultos }\end{array}$ \\
\hline Compreensivo & $\begin{array}{l}\text { Ferreira \& Villemor-Amaral } \\
\qquad(2005)\end{array}$ & Paidéia (Ribeirão Preto) & $\begin{array}{l}\text { Evidências de } \\
\text { validade }\end{array}$ & Adultos \\
\hline Compreensivo & $\begin{array}{l}\text { Villemor-Amaral, Machado } \\
\quad \& \text { Noronha (2009) }\end{array}$ & $\begin{array}{c}\text { Psicologia Ciência e } \\
\text { Profissão }\end{array}$ & Precisão & Adultos \\
\hline Compreensivo & $\begin{array}{l}\text { Villemor-Amaral \& } \\
\text { Machado (2011) }\end{array}$ & Paidéia (Ribeirão Preto) & $\begin{array}{l}\text { Evidências de } \\
\text { validade }\end{array}$ & Adultos \\
\hline Compreensivo & $\begin{array}{l}\text { Franco \& Villemor-Amaral } \\
\text { (2012) }\end{array}$ & Avaliação Psicológica & $\begin{array}{l}\text { Evidências de } \\
\text { validade }\end{array}$ & Adultos \\
\hline Compreensivo & $\begin{array}{l}\text { Franco \& Villemor-Amaral } \\
\qquad(2012 b)\end{array}$ & Psico-USF & $\begin{array}{l}\text { Evidências de } \\
\text { validade }\end{array}$ & Adultos \\
\hline Compreensivo & $\begin{array}{l}\text { Villemor-Amaral \& Cardoso } \\
\text { (2012) }\end{array}$ & Psico/PUCRS & $\begin{array}{l}\text { Evidências de } \\
\text { validade }\end{array}$ & Adultos \\
\hline Compreensivo & $\begin{array}{c}\text { Grazziotin \& Scortegagna } \\
(2012)\end{array}$ & Psicologia: Reflexão e Crítica & $\begin{array}{l}\text { Evidências de } \\
\text { validade }\end{array}$ & Adultos \\
\hline Compreensivo & $\begin{array}{c}\text { Grazziotin \& Scortegagna } \\
\text { (2013) }\end{array}$ & Psico-USF & $\begin{array}{l}\text { Evidências de } \\
\text { validade }\end{array}$ & Adultos \\
\hline Compreensivo & $\begin{array}{l}\text { Villemor-Amaral \& Quirino } \\
\qquad(2013)\end{array}$ & Avaliação Psicológica & $\begin{array}{l}\text { Evidências de } \\
\text { validade }\end{array}$ & Crianças \\
\hline Compreensivo & $\begin{array}{l}\text { Tavella \& Villemor-Amaral } \\
\qquad(2014)\end{array}$ & $\begin{array}{c}\text { Estudos de Psicologia- } \\
\text { Campinas }\end{array}$ & $\begin{array}{l}\text { Evidências de } \\
\text { validade }\end{array}$ & Crianças \\
\hline Compreensivo & $\begin{array}{c}\text { Grazziotin \& Scortegagna } \\
(2016)\end{array}$ & Psico-USF & $\begin{array}{l}\text { Evidências de } \\
\text { validade }\end{array}$ & Adultos \\
\hline Compreensivo & $\begin{array}{l}\text { Villemor-Amaral, Pavan, } \\
\text { Tavella, Cardoso \& Biasi } \\
(2016)\end{array}$ & Paidéia (Ribeirão Preto) & $\begin{array}{l}\text { Evidências de } \\
\text { validade }\end{array}$ & Crianças \\
\hline Compreensivo & $\begin{array}{l}\text { Villemor-Amaral \& Vieira } \\
\qquad(2016)\end{array}$ & Paidéia (Ribeirão Preto) & $\begin{array}{l}\text { Evidências de } \\
\text { validade }\end{array}$ & Crianças \\
\hline Compreensivo & $\begin{array}{c}\text { Gregoleti \& Scortegagna } \\
\text { (2017) }\end{array}$ & Paidéia (Ribeirão Preto) & $\begin{array}{l}\text { Evidências de } \\
\text { validade }\end{array}$ & Idosos \\
\hline Manual Suíço & $\begin{array}{c}\text { Candiani, Souza, Camilo \& } \\
\text { Candiani (2003) }\end{array}$ & $\begin{array}{c}\text { PSIC - Revista de Psicologia } \\
\text { da Vetor Editora }\end{array}$ & $\begin{array}{l}\text { Evidências de } \\
\text { validade }\end{array}$ & Adultos \\
\hline Manual Suíço & Candiani (2004) & $\begin{array}{c}\text { PSIC - Revista de Psicologia } \\
\text { da Vetor Editora }\end{array}$ & Precisão & Adultos \\
\hline Manual Argentino & $\begin{array}{l}\text { Coelho, Bastos, Câmara \& } \\
\text { Landeira-Fernandez (2010) }\end{array}$ & $\begin{array}{c}\text { Estudos de Psicologia- } \\
\text { Campinas }\end{array}$ & $\begin{array}{l}\text { Investigar afetividade } \\
\text { ou cognição }\end{array}$ & Idosos \\
\hline
\end{tabular}




\section{W'INTERACÃO EM ET PSICOLOGIA}

Lucila Moraees Cardoso, Gabriel Vitor Acioly Gomes, Fabio Pinheiro Pacheco e João Lucas Dias Viana

Em seguida, os artigos foram analisados em relação à autoria do trabalho e às instituições de vínculo dos primeiros autores, conforme Tabela 2. A distribuição regional dos primeiros autores de cada artigo foi feita com base na localização das instituições nas quais eles estavam vinculados. Dentre os artigos, somente uma publicação sobre o Zulliger estava fora do eixo Sul-Sudeste, sendo na região Nordeste.

Tabela 2. Informações Sobre a Autoria e Instituição de Origem dos Artigos Com o Teste de Zulliger

\begin{tabular}{|c|c|c|c|}
\hline Categoria & Classificação & Artigos (N) & $\%$ \\
\hline \multirow{3}{*}{ Quantidade de autores } & Individual & 1 & 5,00 \\
\hline & Dois & 15 & 75,00 \\
\hline & Três ou mais & 4 & 20,00 \\
\hline \multirow{2}{*}{ Sexo do $1^{\circ}$ autor } & Feminino & 19 & 95,00 \\
\hline & Masculino & 1 & 5,00 \\
\hline \multirow{4}{*}{ Titulação do $1^{\circ}$ autor } & Especialista & 3 & 15,00 \\
\hline & Mestre & 7 & 35,00 \\
\hline & Doutor & 4 & 20,00 \\
\hline & Pós-doutor & 6 & 30,00 \\
\hline \multirow{9}{*}{$\begin{array}{l}\text { Instituição de origem do } 1^{\circ} \\
\text { autor }\end{array}$} & $\begin{array}{l}\text { Universidade São Francisco } \\
\text { (Particular) }\end{array}$ & 8 & 40,00 \\
\hline & $\begin{array}{l}\text { Universidade de Passo Fundo } \\
\text { (Particular) }\end{array}$ & 4 & 20,00 \\
\hline & $\begin{array}{l}\text { Universidade Federal de Minas } \\
\text { Gerais (Pública) }\end{array}$ & 2 & 10,00 \\
\hline & $\begin{array}{l}\text { Centro Universitário Nossa } \\
\text { Senhora do Patrocínio }\end{array}$ & 1 & 5,00 \\
\hline & $\begin{array}{l}\text { Universidade Federal do Rio } \\
\text { Grande do Norte (Pública) }\end{array}$ & 1 & 5,00 \\
\hline & $\begin{array}{l}\text { Universidade Federal Fluminense } \\
\text { (Pública) }\end{array}$ & 1 & 5,00 \\
\hline & $\begin{array}{l}\text { Escola para Surdos Frei Pacífico e } \\
\text { do Centro Interdisciplinar de } \\
\text { Saúde Mental - CISAME } \\
\text { (Particular) }\end{array}$ & 1 & 5,00 \\
\hline & $\begin{array}{l}\text { Pontifícia Universidade Católica } \\
\text { (Particular) }\end{array}$ & 1 & 5,00 \\
\hline & $\begin{array}{l}\text { Université de Picardie Jules } \\
\text { Vernes (Estrangeira) }\end{array}$ & 1 & 5,00 \\
\hline \multirow{6}{*}{ Estado } & São Paulo & 9 & 45,00 \\
\hline & Rio Grande do Sul & 6 & 30,00 \\
\hline & Minas Gerais & 2 & 10,00 \\
\hline & Rio Grande do Norte & 1 & 5,00 \\
\hline & Rio de Janeiro & 1 & 5,00 \\
\hline & Amiens/França & 1 & 5,00 \\
\hline
\end{tabular}




\section{WIINTERACÃO EM ETH PSICOLOGIA}

Ao analisar a Tabela 3 sobre os periódicos e o ano de publicação dos artigos que utilizaram o Teste de Zulliger, verificou-se que a maioria dos artigos foi publicada nos
Lucila Moraees Cardoso, Gabriel Vitor Acioly Gomes, Fabio Pinheiro Pacheco e João Lucas Dias Viana

\begin{tabular}{|c|c|c|c|}
\hline Categoria & Classificação & Artigos (N) & $\%$ \\
\hline \multirow{9}{*}{ Periódico } & Paidéia (Ribeirão Preto) & 5 & 25,00 \\
\hline & Psico-USF & 4 & 20,00 \\
\hline & $\begin{array}{l}\text { PSIC - Revista de Psicologia } \\
\text { da Vetor }\end{array}$ & 3 & 15,00 \\
\hline & Avaliação Psicológica & 2 & 10,00 \\
\hline & $\begin{array}{l}\text { Estudos de Psicologia- } \\
\text { Campinas }\end{array}$ & 2 & 10,00 \\
\hline & Psicologia Ciência e Profissão & 1 & 5,00 \\
\hline & Psicologia: Reflexão e Crítica & 1 & 5,00 \\
\hline & Psicologia: Teoria e Pesquisa & 1 & 5,00 \\
\hline & Psico/PUCRS & 1 & 5,00 \\
\hline \multirow{11}{*}{ Ano } & 2003 & 3 & 15,00 \\
\hline & 2004 & 1 & 5,00 \\
\hline & 2005 & 1 & 5,00 \\
\hline & 2009 & 2 & 10,00 \\
\hline & 2010 & 1 & 5,00 \\
\hline & 2011 & 1 & 5,00 \\
\hline & 2012 & 4 & 20,00 \\
\hline & 2013 & 2 & 10,00 \\
\hline & 2014 & 1 & 5,00 \\
\hline & 2016 & 3 & 15,00 \\
\hline & 2017 & 1 & 5,00 \\
\hline
\end{tabular}

periódicos Paidéia (Ribeirão Preto) e Psico-USF. Além disso, nota-se um pico de publicações em 2012, com quatro artigos (20\%) publicados neste ano.
Buscou-se também analisar a relação entre as instituições onde os estudos foram desenvolvidos e o sistema interpretativo do teste de Zulliger, conforme Tabela 4. Houve 14 artigos (70\%) que usaram o ZSC de Villemor-Amaral e Primi (2009) e três (15\%) que utilizaram pelo sistema de interpretação Klopfer de Vaz (1998).
Os estudos que utilizaram o ZSC foram realizados em dois estados brasileiros, a saber, São Paulo e Rio Grande do Sul, e em uma instituição internacional utilizando-se de amostra com brasileiros. As pesquisas com a forma interpretativa Klopfer do instrumento estavam distribuídos nos estados do Rio Grande do Norte e Rio Grande do Sul. 


\section{H NTERAC̄öEM IT PSICOLOGIA}

Tabela 4. Relação Entre Sistema Adotado e Instituição de Origem do $1{ }^{\circ}$ Autor dos Artigos

\begin{tabular}{llcc}
\hline Sistema Interpretativo & Instituição de origem do 1 ${ }^{\circ}$ autor & Artigos (N) & \% \\
\hline \multirow{3}{*}{ Sistema compreensivo } & Universidade São Francisco & 8 & 40,00 \\
\cline { 2 - 4 } & Universidade de Passo Fundo & 4 & 20,00 \\
\cline { 2 - 4 } & $\begin{array}{l}\text { Centro Universitário Nossa Senhora do } \\
\text { Patrocínio }\end{array}$ & 1 & 5,00 \\
\cline { 2 - 4 } & Université de Picardie Jules Vernes & 1 & 5,00 \\
\hline \multirow{3}{*}{ Klopfer } & $\begin{array}{l}\text { Universidade Federal do Rio Grande do } \\
\text { Norte }\end{array}$ & 1 & 5,00 \\
\cline { 2 - 4 } & $\begin{array}{l}\text { Escola para Surdos Frei Pacífico e do } \\
\text { Centro Interdisplinar de Saúde Mental - } \\
\text { CESAME }\end{array}$ & 1 & 5,00 \\
\cline { 2 - 4 } & $\begin{array}{l}\text { Pontifícia Universidade Católica do Rio } \\
\text { Grande do Sul }\end{array}$ & 1 & 5,00 \\
\hline Manual Suiço de 1957 & Universidade Federal de Minas Gerais & 2 & 10,00 \\
\hline Manual Argentino de & Universidade Federal Fluminense & 1 & 5,00 \\
\hline
\end{tabular}

A seguir será apresentada uma breve síntese de cada um dos estudos que utilizaram versões do Zulliger indicadas para uso no Brasil. Assim, primeiramente serão descritos os que adotaram o Sistema Klopfer e posteriormente os do ZSC.

Angelini e Oliveira (2003) investigaram a possibilidade de avaliação psicológica de pessoas surdas a partir do Zulliger. 0 instrumento foi aplicado em dez pessoas com perdas profundas de audição, que cursavam ou já haviam cursado o Ensino Médio. As instruções foram traduzidas para a Língua Brasileira de Sinais (LIBRAS); observou-se que os participantes demonstraram boa capacidade e desempenho, carências afetivas, dificuldades de relacionamento empático com os outros e características depressivas. As autoras concluem que é possível usar o Zulliger com surdos, por psicólogos com fluência em LIBRAS.

Montes e Vaz (2003) objetivaram constatar as condições afetivo-emocionais em mulheres com síndrome pré-menstrual (SPM). A amostra foi constituída por dois grupos, um de 25 mulheres com SPM e outro de 18 mulheres sem SPM. Os resultados indicaram que as mulheres com síndrome pré-menstrual reagem emocionalmente de forma mais intensa e têm tendência a perda do controle emocional, comparadas ao grupo sem sintomas pré-menstruais.

Rodrigues e Alchieri (2009) investigaram a manifestação da afetividade em 70 pessoas com Síndrome de Down. Observou-se que as pessoas com Síndrome de Down expressam sua afetividade mediante características positivas e negativas, tal como crianças que não tenham a síndrome.
A fim de buscar evidências de validade para o ZSC no contexto de seleção de profissionais, Ferreira e Villemor-Amaral (2005) correlacionaram os protocolos do Zulliger com um questionário de Avaliação de Desempenho de 86 colaboradores que passaram por um processo de seleção. As autoras constataram a presença de correlações positivas e significativas do Zulliger com as avaliações de desempenho, apresentando indicadores de validade para o uso do Zulliger no processo de seleção de profissionais da área de exatas.

Villemor-Amaral et al. (2009) averiguaram a precisão teste-reteste do ZSC. 0 instrumento foi administrado individualmente em 25 estudantes de teologia no interior de São Paulo com intervalo de 5 meses entre o teste e o reteste. Os 16 principais indicadores de componentes cognitivos, afetivos e de relacionamento interpessoal foram submetidos às estatísticas descritivas e à correlação de Pearson. Dez indicadores alcançaram índices de precisão satisfatórios (entre 0,60-0,99); quatro tiveram uma precisão moderada (entre 0,40-0,60) e dois não tiveram níveis de significância aceitáveis. Os resultados indicaram boa precisão temporal do instrumento.

Villemor-Amaral e Machado (2011) verificaram se os indicadores de depressão que compõem a Constelação de Depressão (DEPI) do Rorschach no Sistema Compreensivo (SC) auxiliam no diagnóstico de depressão por meio do Zulliger SC. Participaram do estudo 27 pacientes com depressão e 27 não pacientes. As variáveis da constelação DEPI com valores significativos foram FD + V, Sum-SH, Índice-egocentricidade, $\mathrm{CF}+\mathrm{C}<\mathrm{FC}$, Determinantes-mistos e Intelectualização. As 


\section{M* INTERACÃO EM L PSICOLOGIA}

pesquisadoras concluíram que o ZSC é um instrumento que pode contribuir no diagnóstico da depressão.

$\mathrm{Na}$ busca de evidências de validade para uso do ZSC no contexto da saúde mental, Franco e Villemor-Amaral (2012) analisaram 141 protocolos do teste de Zulliger, dos quais 46 eram de pacientes psiquiátricos com diagnóstico confirmado pela SCID-I e 95 de não-pacientes. Após compararem os indicadores das constelações de psicopatologia entre os dois grupos, concluíram que as constelações de psicopatologia do Rorschach pelo Sistema Compreensivo não podem ser transpostas para o Zulliger. Deste modo, o ZSC é mais eficaz para compreensão de aspectos da dinâmica de personalidade que independem de quadros psicopatológicos, auxiliando melhor na compreensão do funcionamento psíquico de uma pessoa do que na atribuição de um diagnóstico nosográfico.

Franco e Villemor-Amaral (2012b) verificaram a validade incremental das Pirâmides Coloridas de Pfister e do ZSC para compreensão da personalidade de dependentes químicos que estavam em tratamento de desintoxicação da droga em centros especializados no Brasil ou na França. Os dois instrumentos foram avaliados pela perspectiva da psicopatologia fenômeno-estrutural. Os resultados indicaram que o Zulliger e o Pfister, quando aplicados de forma associada e interpretados pelo método fenômeno-estrutural, mostraram-se eficientes para conhecer as vivências de espaço e tempo dos sujeitos.

Villemor-Amaral e Cardoso (2012) buscaram por evidências de validade convergente para a classificação do Tipo de Vivência (EB) do Sistema Compreensivo no método de Rorschach e de Zulliger. As correlações dos 51 protocolos de adultos que responderam o Zulliger e o Rorschach evidenciaram que a diferença nos estímulos oferecidos entre as duas técnicas torna menos confiável à definição do Tipo de Vivência no Zulliger segundo os parâmetros do Rorschach. A inconsistência observada sugere a necessidade de estabelecer novas proporções entre $\mathrm{M}$ e WSumC no Zulliger para definir o Tipo de Vivência. Além disso, talvez haja a necessidade de alterar as instruções do Zulliger, estimulando um número maior de resposta dadas a cada prancha, o que naturalmente demandaria novas pesquisas de padronização do Zulliger.

Grazziotin e Scortegagna (2012) verificaram evidências de validade das variáveis de relacionamento do ZSC, por meio da correlação entre o Zulliger e o Inventário de Habilidades Sociais (IHS) de 19 funcionários que trabalhavam diretamente com o público em um supermercado. No ano seguinte e com uma proposta semelhante, Grazziotin e Scortegagna (2013) buscaram evidências de validade das variáveis de relacionamento e produtividade do Zulliger com o IHS. Nesse estudo, foram administrados os instrumentos em 40 sujeitos com idade entre 18 e 43 anos. Os resultados corroboraram o estu-
Lucila Moraees Cardoso, Gabriel Vitor Acioly Gomes, Fabio Pinheiro Pacheco e João Lucas Dias Viana do anterior das autoras e o aumento no número de participantes possibilitou confirmar as correlações que no estudo de 2012 haviam sido marginalmente significativas. Além disso, o número de respostas ( $R$ ) não obteve correlação significativa do ponto de vista estatístico com os fatores do IHS, mas qualitativamente denotou conexão. Deste modo, os resultados desses dois estudos das autoras contribuíram para evidências de validade do ZSC.

Villemor-Amaral e Quirino (2013) demonstraram evidências de validade do ZSC por meio da correlação entre os tipos de resposta de cor (FC, CF e C) no Zulliger com o aspecto formal (Tapetes, Formações e Estruturas) no teste de Pfister. Participaram 60 estudantes de escolas públicas de São Paulo, sendo 30 crianças com 6 anos de idade e 30 com 12 anos. As autoras partiram da hipótese de que crianças maiores teriam maior controle emocional, entretanto o indicador de respostas impulsivas no ZSC (C) se correlacionou com o aumento de Estruturas do Pfister no grupo mais velho. Foi problematizada a possibilidade de esse resultado ser decorrente da puberdade.

Tavella e Villemor-Amaral (2014) buscaram evidências de validade para uso do Zulliger à avaliação da criatividade infantil. Participaram do estudo, 90 crianças que realizaram o Teste de Criatividade Figural Infantil (TCFI) e o ZSC. A partir dos resultados no TCFI foram compostos dois grupos, um com baixo desempenho e outro com elevada criatividade. Foram encontradas diferenças significativas entre os dois grupos nas variáveis movimento, movimento ativo, qualidade formal incomum, resposta popular e número de respostas. As autoras concluíram que o Zulliger é um instrumento interessante para uso com crianças e sugerem que é importante acumular mais estudos de evidências de validade para identificação de potencial criativo.

Grazziotin e Scortegagna (2016) verificaram a validade das variáveis de mediação do ZSC (XA\%, WDA\%, X-\%, X+\%, $\mathrm{Xu}, \mathrm{S}$ - e p). Participaram do estudo 40 adultos residentes no interior do Rio Grande do Sul. O desempenho dos participantes foi comparado com os valores normativos contidos no manual do teste e os resultados apontaram diferenças estatísticas significativas entre os dois grupos. A amostra gaúcha apresentou XA\%, WDA\% e X+\% aumentadas e rebaixamento de $\mathrm{X}-\%$ e $\mathrm{S}-\%$. As autoras atribuíram esses resultados ao valor que os aspectos culturais possuem na construção da personalidade e destacaram a importância da realização de estudos normativos com amostras mais representativas e diversificadas, oriundas das diversas regiões brasileiras.

Villemor-Amaral et al. (2016) avaliaram a dinâmica afetiva e cognitiva de crianças em diferentes fases do desenvolvimento. O ZSC foi aplicado em 103 crianças (38 crianças de 6 anos e 65 de 12 anos) de escolas públicas do interior de São 


\section{H NTERAC̄öEM ET PSICOLOGIA}

Lucila Moraees Cardoso, Gabriel Vitor Acioly Gomes,

Fabio Pinheiro Pacheco e João Lucas Dias Viana
Paulo. Dentre os resultados, as crianças mais novas apresentaram escores acima da média no determinante de cor acromática ( $\left.C^{\prime}\right)$, na qualidade formal FQ-, nos conteúdos para-humanos $[(\mathrm{H})]$, comida $(\mathrm{Fd})$ e anatomia $(\mathrm{An})$ e o código especial de desvio de verbalização (DV). Já as crianças de 12 anos de idade apresentaram aumento dos determinantes de movimento humano (M), sombreamento difuso (FY), sombreamento de textura (FT), forma dimensão (FD) e conteúdo de paisagem (Ls). As autoras salientaram a importância de pesquisas longitudinais para acompanhar as fases do desenvolvimento.

Villemor-Amaral e Vieira (2016) buscaram evidências de validade para o ZSC na avaliação da maturidade para o relacionamento interpessoal [variáveis $\mathrm{H}, \mathrm{Hd},(\mathrm{H}),(\mathrm{Hd}), \mathrm{M}, \mathrm{FC}, \mathrm{CF}, \mathrm{C}$, $A, A d,(A),(A d), G H R, P H R, A G$ e COP] em crianças por meio da comparação entre um grupo com 6 anos e outro de 12 anos de idade. Nas comparações entre os sexos, foi observado que os escores de FC e Ad eram maiores em meninos do que em meninas, enquanto os escores das variáveis $\mathrm{H}$ e $\mathrm{A}$ eram mais frequentes em meninas. Quando os grupos foram comparados quanto a idade, foi observado que a variável $(H)$ era mais frequente no grupo de crianças de 6 anos, enquanto a variável $\mathrm{M}$ apareceu mais no grupo de crianças de 12 anos. Os autores consideraram que o estudo contribuiu substancialmente para a compreensão de como as crianças desenvolvem aspectos relacionados à maturidade nas relações interpessoais, tanto em função do sexo quanto da diferença de idades.

Gregoleti e Scortegagna (2017) investigaram a utilidade do ZSC na avaliação de idosos com Doença Renal Crônica, enfatizando os construtos cognitivos e de relacionamento interpessoal. Participaram do estudo 60 idosos (média de 73 anos) do Rio Grande do Sul divididos em grupo clínico e não clínico. Os resultados das comparações mostraram que, no grupo clínico, os escores das variáveis $\mathrm{Xu} \%, \mathrm{R}, \mathrm{Fd}$ e índice de isolamento eram mais baixos, enquanto os escores para $X-\%$, e $\mathrm{PHR}>\mathrm{GHR}$ eram mais altos, em comparação ao grupo não clínico. Assim, os autores concluíram que idosos com doença renal crônica apresentam decréscimo da produtividade, problemas cognitivos e prejuízos nas relações interpessoais, evidenciando o Zulliger como um importante instrumento no processo de avaliação.

\section{DISCUSSÃO}

As revisões da literatura são importantes por possibilitarem uma compreensão geral de como está a produção científica de uma determinada área (Joly et al, 2010). Assim, a partir dos artigos selecionados foi possível visualizar o desenvolvimento do teste Zulliger no Brasil entre 2003 e 2017, evidenciando aumento na frequência dos estudos nos últimos anos. Primi (2010) e Noronha e Reppold (2010) descreveram os avanços na área de Avaliação Psicológica, destacando o aumento progressivo na produção científica da área. Nota-se, deste modo, que os avanços nos estudos com o Zulliger ocorreram concomitantes aos avanços da área de Avaliação Psicológica no Brasil.

$\mathrm{Na}$ Tabela 2, observou-se a elevada porcentagem de publicações com dois autores (75\%) e que 17 (85\%) dos primeiros autores tinham como titulação mínima o mestrado. Esses dados sugerem que o sistema de coautoria facilita a produção científica, uma vez que essa prática possibilita a troca de conhecimento entre os autores e, consequentemente, o aprofundamento do tema abordado, especialmente no campo de pesquisa de testes (Hutz \& Bandeira, 2003).

Para além disso, sabe-se que os Programas de Pós-graduação são difusores do conhecimento e os principais propulsores de pesquisas, assim potencialmente esses artigos podem ser oriundos de dissertações ou teses, justificando essa elevada incidência de trabalhos com dois autores. Nesse sentido, vale lembrar que os autores do manual do Zulliger pelo Sistema Compreensivo são vinculados ao Programa de Pós-graduação em Psicologia da Universidade São Francisco, localizado em São Paulo, e o autor do Zulliger pelo Sistema Klopfer aposentou-se como professor no Programa de Pósgraduação da Pontifícia Universidade Católica do Rio Grande do Sul (PUC-RS).

Ao verificar a distribuição geográfica das instituições de filiação dos pesquisadores (Tabela 2), ficou evidente uma predominância do Sudeste, seguido pelo Sul. Dados semeIhantes foram obtidos nas pesquisas de Joly et al. (2007) e Joly et al. (2010), nas quais verificaram-se que a maioria das produções na área de avaliação psicológica são de universidades da região sudeste, em especial das particulares, sobressaltando as instituições que já são conhecidas por desenvolver pesquisas na área.

Ao analisar, na Tabela 3, em que periódicos esses artigos foram publicados, houve destaque às revistas Paidéia (Ribeirão Preto) e Psico-USF. Ambas as revistas possuem boa avaliação pelo Sistema de Avaliação de Periódicos da CAPES na área de avaliação "psicologia", sendo classificadas como A1 e $\mathrm{A} 2$, respectivamente.

Também na Tabela 3, notou-se que dos sete estudos com o Zulliger publicados no período entre 2003 e 2009, três envolviam o uso do Sistema Klopfer, publicado no Brasil por Vaz (1998) e atualizado recentemente por Vaz e Alchieri (2016), e dois utilizaram o ZSC (Villemor-Amaral \& Primi, 2009). Esses números inverteram a partir de 2010 de modo que das 13 produções posteriores, 12 foram com o Sistema Compreensivo. A publicação do manual do ZSC para adultos, em 2009, 


\section{W NTERACÃO EM ETS PSICOLOGIA}

pode ser um dos principais motivos desse aumento, pois era preciso buscar evidências de validade para o sistema recémpublicado. 0 fato de 17 (85\%) dos trabalhos terem o objetivo de estudar as qualidades psicométricas do Zulliger reforça a ideia citada.

$\mathrm{Na}$ Tabela 4, verificou-se que dos 14 estudos que utilizaram o Sistema Compreensivo, oito foram realizados por autores vinculados a Universidade São Francisco (USF), quatro a Universidade de Passo Fundo, um ao Centro Universitário Nossa Senhora do Patrocínio e um a uma instituição francesa. Todos os primeiros autores que não eram diretamente vinculados à USF tiveram vínculo com o programa de pós-graduação da universidade em algum momento de sua trajetória acadêmica. Esses dados mostram a USF como um polo de estudo e disseminação de pesquisa com este método. Conforme Joly et al. (2010), essa universidade tem como referência um programa de pós-graduação stricto sensu e é uma das que mais produz na área de avaliação psicológica.

O fato da maioria das pesquisas com o ZSC buscar por qualidades psicométricas (Pasquali, 2010; Miguel, 2017) contribui para os avanços do método, em especial, ao verificar o interesse de buscar evidências de validade para diferentes faixas etárias e contextos. Nota-se que das pesquisas conduzidas uma indicou boa precisão temporal (Villemor-Amaral et al., 2009), três indicaram boas evidências de validade para uso no contexto organizacional (Ferreira \& Villemor $\neg$ Amaral, 2005; Grazziotin \& Scortegagna, 2012; Grazziotin \& Scortegagna, 2013), quatro para uso com crianças (Villemor-Amaral \& Quirino, 2013; Tavella \& Villemor-Amaral, 2014; VillemorAmaral et al., 2016; Villemor-Amaral \& Vieira, 2016) e os demais avaliaram a validade de alguns indicadores do instrumento (Villemor-Amaral \& Machado, 2011; Franco \& Villemor-Amaral, 2012; Franco \& Villemor-Amaral, 2012b; Villemor-Amaral \& Cardoso, 2012; Grazziotin \& Scortegagna, 2016; Gregoleti \& Scortegagna, 2017).

Grazziotin e Scortegagna (2016b) descreveram um panorama restrito para uso do instrumento na medida em que a maioria das pesquisas foi realizada com participantes adultos do estado de São Paulo. Ainda que essa crítica se faça atual, por meio da Tabela 1 verifica-se que nos últimos três anos houve investimento no sentido de diversificar os estudos para o público infantil (Villemor-Amaral et al., 2016; Villemor-Amaral \& Vieira, 2016) e idosos (Gregoleti \& Scortegagna, 2017). Parte desses estudos apontaram a demanda por mais pesquisas e a necessidade de construção de tabelas normativas para diferentes faixas etárias (VillemorAmaral et al., 2016 e Villemor-Amaral \& Vieira, 2016).

Além disso, há que se expandirem os estudos para diferentes regiões geográficas do Brasil. Tal relevância decorre da necessidade de considerar a grande extensão territorial do
Lucila Moraees Cardoso, Gabriel Vitor Acioly Gomes,

Fabio Pinheiro Pacheco e João Lucas Dias Viana
Brasil, devendo os instrumentos adequar-se às diversas realidades a fim de que atendam às normativas das qualidades psicométricas (Noronha \& Alchieri, 2002; Serafini et al., 2017, CFP, 2003).

Os avanços nos estudos psicométricos com o Zulliger sem dúvidas representam uma conquista para área. É preciso, entretanto, reforçar a escassez e a importância de que também sejam conduzidos estudos de caráter idiográfico do método, tal como defendido por Fensterseifer e Werlang (2008) e Villemor-Amaral e Pasqualini (2006) ao tratarem dos estudos com os métodos projetivos (Meyer \& Kurtz, 2006; Rietzler, 2006; Miguel, 2014; Cardoso \& Villemor-Amaral, 2017) de um modo geral.

Ademais, esta revisão de literatura ateve-se à compreensão dos artigos do Zulliger com brasileiros, restringindo-se a um curto período de tempo. Nessa perspectiva, sugere-se que pesquisas futuras possam considerar a produção científica internacional, verificando, assim, os avanços desse método em outros países e, com bases nas produções destes, discutir e fortalecer as pesquisas realizadas no Brasil.

\section{CONTRIBUIÇÃO DE CADA AUTOR}

Todos os autores participaram da busca e análise dos artigos, bem como da escrita e revisão do trabalho.

\section{DECLARAÇÃO DE CONFLITOS DE INTERESSES}

Os autores declaram que não há conflitos de interesses no presente artigo.

\section{REFERÊNCIAS}

Angelini, S. N., \& Oliveira, R. V. (2003). Aplicação do teste verbal Zulliger (forma individual) em pessoas surdas. Psic, 4(1), pp. 82-93

Candiani, D. M. de A. (2004). Precisão do Teste de Zulliger. Psic, 5(1), 16 21.

Candiani, D. M. de A., Souza, A. M. R. de., Camilo, D., \& Candiani, T. M. (2003). Estudo da validade de um método projetivo: Teste de Zulliger - por meio de parâmetros psicométricos. Psic, 4(2), pp. 36-43.

Cardoso, L. M., \& Villemor-Amaral, A. E. (2017). Critérios de Cientificidade dos métodos projetivos. Em M. R. C. Lins \& J. C. Borsa (Eds.), Avaliação psicológica: Aspectos teóricos e práticos (pp. 159-172). Petrópolis, RJ: Vozes Editora.

Coelho, C. L. M., Bastos, C. L., Camara, F. P., \& Landeira-Fernandez, J. (2010). A influência do gênero e da escolaridade no diagnóstico de demência. Estudos de psicologia (Campinas), 27(4), pp. 448-456. http://dx.doi.org/ 10.1590/ 


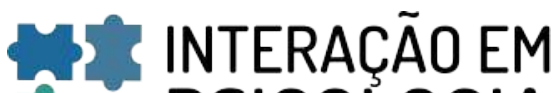 ET PSICOLOGIA}

\section{S0103-166X2010000400003}

Conselho Federal de Psicologia. (2018). Resolução n CFP 009/2018. Brasília, DF.

Fensterseifer, L., \& Werlang, B. S. G. (2008). Apontamentos sobre o status científico das técnicas projetivas. Em A. E. Villemor-Amaral \& B. S. G. Werlang (Eds.), Atualizações em métodos projetivos para avaliação psicológica (pp. 1533). São Paulo, SP: Casa do Psicólogo.

Ferreira, M. E. A., \& Villemor $\neg$ Amaral, A. E. de. (2005). 0 teste de Zulliger e avaliação de desempenho. Paidéia (Ribeirão Preto), 15(32), pp. 367-376. http://dx.doi.org/10.1590/ S0103-863X2005000300006

Franco, R. da R. C., \& Villemor-Amaral, A. E. de. (2012). O Zulliger e as constelações do Rorschach no sistema compreensivo. Avaliação Psicológica, 11(1), pp. 141-152.

Franco, R. da R. C., \& Villemor-Amaral, A. E. de. (2012b). Validade incremental do Zulliger e do Pfister no contexto da toxicomania. Psico-USF, 17(1), pp. 73-83. http://dx.doi.org/ 10.1590/S1413-82712012000100009

Grazziotin, J. B. di D., \& Scortegagna, S. A. (2012). Zulliger e habilidade social: Evidências de validade no contexto empresarial. Psicologia: Reflexão e Crítica, 25(1), pp. 69-78. http://dx.doi.org/10.1590/S0102-79722012000100009

Grazziotin, J. B. di D., \& Scortegagna, S. A. (2013). Relacionamento interpessoal, produtividade e habilidades sociais: um estudo correlacional. Psico-USF, 18(3), pp. 491-500. http://dx.doi.org/10.1590/S1413-82712013000300015

Grazziotin, J. B. di D., \& Scortegagna, S. A. (2016). Mediação no Zulliger: Evidências de validade em amostra de não pacientes. Psico-USF, 21(1), pp. 63-72. doi: 10.1590/1413-

Grazziotin, J. B. di D., \& Scortegagna, S. A. (2016b). Revisão de pesquisas brasileiras sobre o teste de Zulliger publicadas em artigos. Avaliação Psicológica, 15(2), pp. 227-235. http://dx.doi.org/10.15689/ap.2016.1502.11

Gregoleti, V., \& Scortegagna, S. A. (2017). The Zulliger-CS in elderly on hemodialysis and the relationship between external variables. Paidéia (Ribeirão Preto), 27(66), pp. 4350. http://dx.doi.org/10.1590/1982-43272766201706

Hutz, C. S., \& Bandeira, D. R. (2003). Avaliação psicológica no Brasil: Situação atual e desafios para o futuro. Em O. H. Yamamoto \& V. V. Gouveia (Eds.), Construindo a psicologia brasileira: Desafios da ciência e prática psicológica (pp. 261-275). São Paulo, SP: Casa do Psicólogo.

Joly, M. C. R. A., Silva, M. C. R. da, Nunes, M. F. O., \& Souza, M. S. de. (2007). Análise da produção científica em painéis dos congressos brasileiros de avaliação psicológica. Avaliação Psicológica, 6(2), pp. 239-252.

Joly, M. C. R. A., Teixeira, T. C., Berberian, A. de A., \& Andrade, R. G. de. (2010). Análise de teses e dissertações em avaliação psicológica disponíveis na BVS-PSI Brasil. Psicologia 82712016210106
Lucila Moraees Cardoso, Gabriel Vitor Acioly Gomes, Fabio Pinheiro Pacheco e João Lucas Dias Viana
Meyer, G. J., \& Kurtz, J. E. (2006). Advancing personality assessment terminology: Time to retire "objective" and "projective" as personality test descriptors. Journal of Personality Assessment, 87(3), pp. 223-225. http:// dx.doi.org/ 10.1207/s15327752jpa8703_01

Miguel, F. K. (2014). Mitos e verdades no ensino de técnicas projetivas. Psico-USF, 19(1), pp. 97-106. http://dx.doi.org/ 10.1590/S1413-82712014000100010

Miguel, F. K. (2017). Padronização e interpretação dos resultados. Em M. R. C. Lins \& J. C. Borsa (Eds.), Avaliação psicológica: Aspectos teóricos e práticos (pp.127-136). Rio de Janeiro, RJ: Editora Vozes.

Montes, R. M., \& Vaz, C. E. (2003). Condições afetivoremocionais em mulheres com síndrome pré-menstrual através do ZᄀTeste e do IDATE. Psicologia: Teoria e Pesquisa, 19(3), pp. 261 -267. http://dx.doi.org/10.1590/S010237722003000300008

Noronha, A. P. P., \& Alchieri, J. C. (2002). Reflexões sobre os instrumentos de avaliação psicológica. Em R. Primi (Ed.), Temas em avaliação psicológica (pp. 19-36). São Paulo, SP: Casa do Psicólogo.

Noronha, A. P. P., \& Reppold, C. T. (2010). Considerações Sobre a Avaliação Psicológica no Brasil. Psicologia Ciência e Profissão, 30(núm. esp.), pp. 192-201. http://dx.doi.org/ 10.1590/S1414-98932010000500009

Pasquali, L. (2010). Instrumentação psicológica: Fundamentos e práticas. Porto Alegre: Artmed.

Primi, R. (2010). Avaliação psicológica no Brasil: Fundamentos, situação atual e direções para o futuro. Psicologia: Teoria e Pesquisa, 26(núm. esp.), pp. 25-35. http:// dx.doi.org/ 10.1590/S0102-37722010000500003

Rietzler, B. (2006). Aplicações culturais do Rorschach, testes de apercepção e desenho de figuras. Em N. Abreu \& D. M. Amparo (Eds.), Métodos projetivos: Instrumentos atuais para a investigação psicológica e da cultura. São Paulo, SP: Vetor.

Rodrigues, E. C., \& Alchieri, J. C. (2009). Avaliação das características de afetividade em crianças e jovens com síndrome de Down. Psico-USF, 14(1), pp. 107-116.

Serafini, A. J., Budzyn, C. de S., \& Fonseca, T. L. R. (2017). Tipos de testes características e aplicabilidade. Em M. R. C. Lins \& J. C. Borsa (Eds.), Avaliação Psicológica: Aspectos Teóricos e Práticos (pp. 56-75). Rio de Janeiro, RJ: Editora Vozes.

Tavella, R. R., \& Villemor-Amaral, A. E. de. (2014). O teste de Zulliger-SC: Avaliação da criatividade em crianças. Estudos de Psicologia (Campinas), 31(4), pp. 489-497. http:// dx.doi.org/ 10.1590/0103-166X2014000400003

Vaz, C. E. (1998). Zulliger: A técnica de Zulliger forma coletiva. São Paulo, SP: Casa do Psicólogo. 


\section{-4: INTERACÃO EM L* PSICOLOGIA}

Vaz, C. E., \& Alchieri, J. C. (2016). Z-Teste coletivo e individual: Técnica de Zulliger. São Paulo, SP: Hogrefe.

Villemor-Amaral, A. E. de, \& Cardoso, L. M. (2012). Validade convergente do tipo de vivência (EB) no teste de Zulliger/ SC. Psico/PUCRS, 43(1), pp. 109-115.

Villemor-Amaral, A. E. de, \& Machado, M. A. dos S. (2011). Indicadores de depressão do Zulliger no Sistema Compreensivo (ZSC). Paidéia, 21(48), pp. 21-27. http://dx.doi.org/ 10.1590/S0103-863X2011000100004

Villemor-Amaral, A. E. de, \& Primi, R. (2009). Teste de Zulliger no sistema compreensivo - ZSC: Forma individual. São Paulo, SP: Casa do Psicólogo.

Villemor-Amaral, A. E. de, \& Quirino, G. de S. (2013). Estudo comparativo entre indicadores afetivos das técnicas de Pfister e Zulliger. Avaliação Psicológica, 12(1), pp. 1-7.

Villemor-Amaral, A. E. de, \& Vieira, P. G. (2016). Zulliger (CS) in assessing the relational maturity of children. Paidéia (Ribeirão Preto), 26(65), pp. 369-376. http://dx.doi.org/ $10.1590 / 1982-43272665201601$
Lucila Moraees Cardoso, Gabriel Vitor Acioly Gomes, Fabio Pinheiro Pacheco e João Lucas Dias Viana

Villemor-Amaral, A. E. de, Machado, M. A. dos S., \& Noronha, A. P. P. (2009). O Zulliger no sistema compreensivo: Um estudo de fidedignidade. Psicologia Ciência e Profissão, 29(4), pp. 656-671. http://dx.doi.org/10.1590/S141498932009000400002

Villemor-Amaral, A. E. de, Pavan, P. M. P., Tavella, R. R., Cardoso, L. M., \& Biasi, F. C. (2016). Validity evidence of the ZTest-SC for use with children. Paidéia (Ribeirão Preto), 26(64), pp. 199-206. http://dx.doi.org/10.1590/198243272664201607

Villemor-Amaral, A. E., de \& Pasqualini-Casado, L. (2006). A cientificidade das técnicas projetivas em debate. PsicoUSF, 11(2), pp. 185-193. http://dx.doi.org/10.1590/S141382712006000200007

Data de submissão: 10/03/2016 Primeira decisão editorial: 16/11/2017 Aceite em 15/02/2018 